PhD Thesis

\title{
IMMUNOPHENOTYPIC MARKER ANAL YSIS OF HAIRY CELL LEUKEMIA IN PARAFFIN-EMBEDDED BONE MARROW TREPHINE BIOPSIES
}

\author{
Judit Tóth-Lipták MD
}

Laboratory of Tumor Pathology and MolecularDiagnostics, Szeged, Hungary 


\section{List of fullpapersbackgroundforthethesis:}

I.Krenács L, Tóth-Lipták J, Demeter J, Piukovics K, Borbényi Z, Gogolák P, Sári E, Bagdi E (2013) Monoclonalantibody HBME-1 reactswith a minor subset of B cellswithvilloussurface and can be usefulinthediagnosis of hairycellleukemia and otherindolentlymphoproliferations of villous B lymphocytes. VirchowsArch. 463:787-794. doi: 10.1007/s00428-013-1490-52013

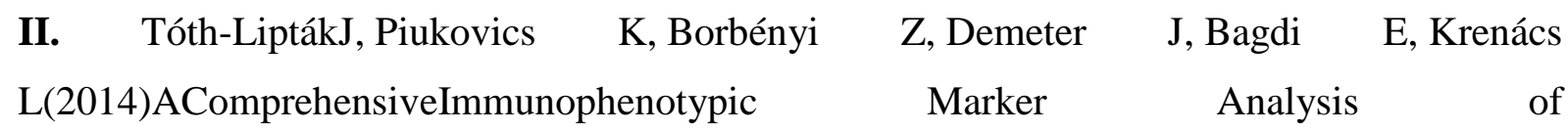
HairyCellLeukemiainParaffin-EmbeddedBoneMarrowTrephineBiopsies-A TissueMicroarrayStudy.PatholOncol Res. Jun 6. [Epubahead of print] 


\section{Introduction}

Hairy cell leukemia (HCL) is a malignant B celllymphoproliferation characterized by pancytopenia, splenomegaly, circulating cells with 'hairy' projections, as well as bone marrow (BM) and splenic involvement. By flow cytometry, the neoplastic cells exhibit a unique immunophenotypic profile, showing 'pan B cell antigen' (e.g. CD19, CD20, and CD22) positivity with consistent co-expression of CD11c, CD25, CD103, and CD123 [Hiba!

A hivatkozási forrás nem található.-Hiba! A hivatkozási forrás nem található.]. There are some indolent B cell lymphoma entities, including hairy cell leukemia variant (HCL-v), splenic diffuse red pulp small B cell lymphoma (SDRPL), and splenic B cell marginal zone lymphoma (SMZL), which can clinically or morphologically mimic HCL, but, in contrast to it, they are unresponsive to interferon- $\alpha$ - or purine analog-containing treatment protocols.Due to the typically low number of circulating tumor cells, as well as a 'dry tap' at BM aspiration caused by HCL-associated reticulin fibrosis, the diagnosis is often based on the histopathological assessment of BM trephine biopsies. There is a broad range of antibodies that are reported to be applicable in fixed and paraffin-embedded HCL samples, including DBA.44, tartrate-resistant acid phosphatase (TRAP), cyclin D1, CD11c, T-bet, and annexin A1 (ANXA1). Nowadays, a BRAF V600E mutation-specific mouse monoclonal antibody, anti-phospho-ERK1/2 (pERK) as well as anti-CD103 rabbit monoclonal antibody have been reported as helpful novel markers for paraffin-embedded HCL samples.

\section{Aims of the work}

I. Evaluation of monoclonal antibody to Hector Battiforamesothelial epitope-1 (HBME1) as a diagnostic marker for HCL and its mimics.

II. Comprehensive analysis of the diagnostic usefulness of various HCL markers (CD11c, CD25, CD68, CD103, CD123, CD200, annexin A1, cyclin D1, DBA.44, HBME-1, phospho-ERK1/2, TRAP, and T-bet) currentlyavailablefor paraffin embedded sections. 
III.We also aimed to define an optimal panel of immunohistochemical stains necessary to reliably differentiate HCL from other villous and non-villous small B cell lymphomas in BM trephine biopsies.

\section{Materials and methods}

\section{Patients and tissue samples}

Cases for the studies were retrieved from the files of the Laboratory of Tumor Pathology and Molecular Diagnostics, Szeged, Hungary from 2001 to 2013. The first study included non-neoplastic bone marrow $(n=15)$, lymph node $(n=12)$, splenectomy $(n=13)$, tonsil $(n=7)$ and gastric biopsy $(n=3)$ samples, in addition to a series of malignant lymphoproliferations ( $\mathrm{n}=427)$, including HCL $(\mathrm{n}=72)$, HCL variant (HCL-v) $(\mathrm{n}=13)$, splenic diffuse red pulp small B cell lymphoma (SDRPL) $(n=8)$, splenic B cell marginal zone lymphoma (SMZL) $(n=59)$, indolent splenic B cell lymphoma, not further classifiable on bone marrow morphology $(\mathrm{SBCL})(\mathrm{n}=37)$, nodal $(\mathrm{n}=30)$ and extranodal $(\mathrm{n}=21)$ marginal zone lymphomas (MZL), other B cell lymphomas $(n=164)$, classical Hodgkin's lymphomas (cHL) $(n=5)$, and T cell lymphomas (TCL) $(\mathrm{n}=18)$.

For comprehensive analysis of HCL markers, 73 cases, including 71 BM trephine biopsy samples and 2 splenectomy specimens were used. The study group comprised HCL $(n=32)$, HCL-v ( $n=4)$, SDRPL $(n=2)$, SMZL $(n=8)$, and SBCL $(n=3)$ cases, moreover BM samples from other small B cell lymphomas, including B cell chronic lymphocytic leukemia (B-CLL) ( $\mathrm{n}=11)$, lymphoplasmacytic lymphoma/Waldenström'smacroglobulinemia (LPL/WM) (n=3), and classical mantle cell lymphoma (MCL) $(\mathrm{n}=10)$ cases.

Each case had been previously diagnosed on the basis of clinical information, histomorphology, flow cytometry, and immunophenotypic characteristics. Diagnosis of SMZL was made on splenectomy specimens showing the characteristic involvement, or on bone marrow trephine biopsies showing the typical interstitial/intrasinusoidal infiltration with larger nodules containing a follicular dendritic cell meshwork. The diagnosis of SDRPL was restricted to cases where splenectomy material was available, showing a characteristic diffuse pattern of involvement of the red pulp. We created SBCL category for those small B cell lymphoma cases with intrasinusoidal bone marrow involvement where the differential diagnosis was narrowed down to indolent splenic B cell lymphoma, but splenectomy has not been performed until the selection into the study, since the bone marrow morphology alone, in most cases, is insufficient to separate SMZL and SDRPL. 
The bone marrow trephine biopsies were fixed for 12-24 hours in neutral buffered formalin supplemented with methanol and glucose (Schaffer's fixative). Since our pilot study has demonstrated significant reductions of HBME-1 staining after acidic decalcification, bone marrow trephines were decalcified in 12,5\% (w/v) EDTA (Sigma-Aldrich) solution (adjusted to $\mathrm{pH} 7.0$ by $\mathrm{cc} \mathrm{NaOH}$ ) at $60^{\circ} \mathrm{C}$ for $16-24$ hours. The other samples were fixed for 24-72 hours in $10 \%(\mathrm{v} / \mathrm{v})$ neutral buffered formalin. Each specimen was routinely embedded into paraffin.

\section{Tissue microarray (TMA) construction}

We constructed TMA blocks with manual Tissue Micro-Array Builder instrument of Histopathology Ltd. (Pécs, Hungary), according to the manufacturer's instructions. Briefly, the recipient paraffin block with 24 holes arranged in four columns and six rows was formed with the TMA Builder. Cores of $2.0 \mathrm{~mm}$ from the donor paraffin blocks were punched out with the Paraffin-Punch-Extractor and were arrayed in the recipient paraffin block. Each sample of interest was represented at least in duplicates, to avoid inadequate amount of sampling.

\section{Immunohistochemistry and evaluation of the reactions}

In our first study immunohistochemical reactions were executed on whole tissue sections and TMA slides.Briefly, $2 \mu \mathrm{m}$-thick paraffin sections were routinely de-waxed, blocked for endogenic peroxidase activities in ethanol containing $1.5 \%(\mathrm{v} / \mathrm{v}) \mathrm{H}_{2} \mathrm{O}_{2}$, and heat-treated in appropriate antigen retrieval buffer solutions using a household electronic pressure cooker (Avair IDA). After protein blocking in TRIS-buffered saline (TBS, $\mathrm{pH} 7.4$ ) containing 5\% (w/v) low fat milk powder, the sections were incubated with the primary antibodies at room temperature for $70 \mathrm{~min}$. In a pilot study, we have tested two distinct monoclonal mouse CD123 antibodies (7G3 and BR4MS), but neither of them has given sufficient sensitivity in HCL cells. To overcome this, a blend of these two antibodies was employed afterwards. For primary goat antibodies, a rabbit anti-goat linker antibody (DAKO, Denmark) was used. Detection was made using Novolink polymer kit (Leica Biosystems/Novocastra), and nuclear staining was carried out with Mayer's hematoxylin. The immunohistochemicalstainings were performed in a 4-channel Freedom Evo liquid handling platform (TECAN, Mannedorf, Switzerland).

To analyze the diagnostic value of the HBME-1 antibody in HCL, HCL-v, SDRPL, SMZL and SBCL cases, the reaction pattern was evaluated in conjunction with CD20, and the results 
were compared against staining with DBA.44. Since up to $10 \%$ HBME-1-positive reactive lymphocytes can occur in hyperplastic lymphoid tissues, an arbitrary cut-off of $20 \%$ staining of CD20-positive Bcells for the HBME-1 or DBA.44 was defined as positive in neoplastic samples. On the basis of findings, sensitivity, specificity, and accuracy were calculated

\section{Results}

\section{HBME-1 in non-neoplastic samples}

In the non-neoplastic samples, scattered $\mathrm{HBME}-1^{+}$sinusoidal and perisinusoidal lymphocytes were constantly depicted in the hyperplastic lymph nodes, showing a strikingly villous cell membrane, and equivalent cells could also be detected in the bone marrow and splenic red pulp.

The positive cells were usually found in low number, but in the splenic specimens with marginal zone hyperplasia, removed due to traumatic rupture or in therapy-resistant idiopathic thrombocytopenic purpura (ITP), up to $10 \%$ red pulp lymphocytes were detected, and a significant proportion of monocytoidB cells in toxoplasma lymphadenitis demonstrated HBME-1 staining. The staining pattern of the HBME-1 and DBA.44 revealed partly overlapping features with the main exception of strong mantle zone staining found only with the DBA.44. Apart from the villous lymphocytes, only a few non-lymphoid cells demonstrated HBME-1 staining. Some follicular dendritic cells and few extrafollicular or chordal reticular cells showed inconsistent weak positivity. In the normal bone marrow samples, an inconsistent low number of interstitial small lymphocytes with villous surface were demonstrated. Scattered early erythroid precursor cells, mostly in erythropoietic hyperplasia also displayed HBME-1 staining.

According to the multicolor immunofluorescence staining we used, the HBME-1 ${ }^{+}$ lymphocytes demonstrated a $\mathrm{CD} 20^{+} / \mathrm{CD} 79 \mathrm{a}^{+} / \mathrm{IgM}^{+} / \mathrm{CD} 35^{\circ} / \mathrm{CD}^{-} \mathrm{B}$ cell phenotype both in lymph node and spleen. In addition, the splenic HBME- ${ }^{+}$B lymphocytes also displayed partial IgD positivity. In non-lymphoid cells, the HBME-1 stained few $\mathrm{CD} 68^{+}$chordalhistiocytes as well as weakly and inconsistently stained a few $\mathrm{CD} 35^{+}$follicular dendritic cells. 


\section{HBME-1 in neoplastic samples}

The HBME-1 staining of tumor cells was exclusively detected in the B cell neoplasms.

Nearly all HCL cases (69/72; 95.8\%) showed HBME-1 positivity, closely matching with the DBA.44 (70/72; 97.2\%). Comparing the proportion of the positive neoplastic cells, no considerable difference was noted for HBME-1 or DBA.44.

In other $B$ celllymphoproliferations of splenic origin, HBME-1 positivity was demonstrated in 5/13 (38.5\%) of HCL-v, 4/8 (50\%) of SDRPL, 7/59 (11.9\%) of SMZL, and $7 / 37(18.9 \%)$ of SBCL cases. Weak and focal HBME-1 positivity was demonstrated in the majority of SMZL and SBCL cases. Higher DBA.44 positivity rate was shown in each of these splenic lymphomas - 11/13 (84.6\%) of HCL-v, 5/8 (62.5\%) of SDRPL, 18/57 (30.5\%) of SMZL, and 12/37 (32.4\%) of SBCL.

Non-splenic MZL cases revealed similar HBME-1 positivity rate $(6 / 51 ; 11.8 \%)$ to SMZL, which was also exceeded by DBA.44 $(2 / 11 ; 18.2 \%)$. Nodal and extranodal marginal zone lymphoma cases showed $13.3 \%$ (4/30) and 9.5\% (2/21) HBME-1 positivity, respectively. The HBME-1 positivity in non-splenic MZL cases was associated with monocytoidB cell morphology. A small minority $(6 / 164 ; 3.7 \%)$ of the other B cell lymphomas demonstrated positivity with the HBME-1. Three cases $(3 / 21 ; 14.3 \%)$ of follicular lymphoma (FL), 2 cases $(2 / 33 ; 6.1 \%)$ of mantle cell lymphoma (MCL) and 1 case $(1 / 41 ; 2.4 \%)$ of diffuse large B cell lymphoma (DLBCL) of probably transformed MZL demonstrated tumor cell positivity with HBME-1.

Apart from scattered reactive lymphocytes and some reticular cells, no HBME-1 positivity was detected in the T cell lymphoma and Hodgkin lymphoma cases tested.

\section{Comprehensive marker analysis of HCL markers}

Most of the immunohistochemical markers tested were highly reactive with neoplastic cells of HCL. The antibodies with surface staining highlighted the 'hairy' appearance of the cell membrane.

Anti-CD20 staining was primarily used to assess the scale of BM involvement, and tumor cell positivity was detected in all but one cases studied. One rituximab-treated MCL case was found to be CD20-negative.

ANXA1 demonstrated strong cytoplasmic and nuclear staining with $100 \%$ positivity rate in HCL cases with apparently $100 \%$ specificity and accuracy, being absent in all non- 
HCL B cell neoplasms. On the other hand, it displayed staining with equivalent strength also in granulocytic series, which caused considerable difficulties in the assessment of quite a number of BM samples.

Phospho-ERK antibody was found to be $100 \%$ sensitive for HCL, moreover showed 97,6\% specificity and $98.6 \%$ accuracy, having been positive in all HCL and negative in all but one small B cell lymphomas other than HCL. This marker revealed nuclear and cytoplasmic staining in HCL cells and also in some non-neoplastic mesenchymal cells in the background, which latter feature could be employed as endogenous positive control in the negative cases.

As expected, both CD11c and CD103antibody revealed 100\% sensitivity in detecting neoplastic cells of HCL. The CD11c/5D11 antibody showed membranous staining with moderate intensity in all but $1 \mathrm{HCL}$ cases as well as weak and mostly focal staining in $1 \mathrm{HCL}$, 2 HCL-v, 1 SDRPL, and 3 B-CLL cases, making up 85.4\% specificity and 91.8\% accuracy. There was an invariable presence of CD11c-positive non-neoplastic dendritic histiocytes and scattered monocytes in BM and splenic samples, which served as internal control of the staining. The CD103/EPR4166(2)antibody exhibited intense membranous staining in all HCL, 3 HCL-v, 1 SDRPL cases as well as weak and focal reactivity in 1 SMZL, providing 87.8\% specificity and $93.2 \%$ accuracy. Apart from the neoplastic HCL cells with membranous positivity, sparse granulocytic cells with weaker membranous and cytoplasmic staining could also be detected using CD103/EPR4166(2).

Immunostaining for CD25 was positive in all HCL cases and negative in all HCL-v, SDRPL, SMZL, and SBCL samples studied. Moreover, 5/11 B-CLL and 5/10 MCL cases were also found to be reactive with the CD25 antibody, leading to $75.6 \%$ specificity and 86.3\% accuracy. Nevertheless, disregarding the B-CLL and MCL cases, the CD25/4C9 antibody had $100 \%$ specificity and accuracy. All positive cases displayed membranous staining with moderate intensity.

All HCL cases showed HBME-1 positivity, highlighting the villous surface of the neoplastic cells. In other small B cell lymphomas, HBME-1 positivity was demonstrated in 2/4 HCL-v, 1/8 SMZL, and 2/2 SDRPL cases, and was found to be negative in all non-splenic small B cell lymphomas, providing $87.8 \%$ specificity and $93.2 \%$ accuracy. Inhomogeneous or partial staining of neoplastic cells was seen in 7 HCL cases, which can cause difficulties in detecting sparse HCL cells.

Cyclin D1 antibody displayed nuclear staining with variable intensity usually in the majority of the HCL cells. Overall it exhibited $73.2 \%$ specificity and $84.9 \%$ accuracy. 
However, excluding MCL cases, the cyclin D1 had very high specificity (96.8\%) and accuracy $(98.4 \%)$ for HCL.

CD68/KP1, CD200, T-bet, and anti-TRAP antibodies were reactive with HCL cells in all cases, but showed positivity in the majority of non-HCL small B cell lymphomas too, which findings determined low specificity for these markers. The CD68/KP1 showed granular cytoplasmic staining with moderate intensity in HCL and other villous lymphoma cases, weak granular staining in B-CLL and LPL cases. It also displayed positive staining in macrophages and myeloid cells which may be disturbing in the evaluation of BM trephines, particularly when the tumor cell burden is low.

The CD200 revealed weak to moderate membranous positivity in HCL, all but 4 HCL-v, SMZL, SDRPL, SBCL, B-CLL as well as LPL/WM cases, and was consistently negative in MCL samples. The T-bet antibody demonstrated strong homogenous nuclear positivity in most HCL, HCL-v, and SDRPL, and weaker staining in the remaining positive cases.

The anti-TRAP immunostaining gave positive staining in all cases of HCL, and was also identified in almost all (38/41) small B cell lymphoma cases, having the lowest specificity (7.3\%) and accuracy (47.9\%) in all. The anti-TRAP antibody had weak staining in LPL/WM and B-CLL cases with increasing expression in the proliferation centers and equivalent strong cytoplasmic staining in both HCL and remaining small B cell lymphoma cases.

All but one (96.9\%) HCL cases were found to be positive with DBA.44, showing predominantly cytoplasmic staining. Of 15 cases of HCL-v, SDRPL, SMZL, and SBCL, 11 were positive (73.3\%), however, all B-CLL, LPL/WM and MCL cases were consistently DBA.44-negative.

The CD123 antibody mixture showed the overall lowest sensitivity, being positive only in $22 / 32(68.8 \%)$ of HCL cases, nevertheless its reactivity was $100 \%$ specific and $86.3 \%$ accurate. The positive cells exhibited membranous staining. Apart from HCL cells, scattered monocytes and plasmacytoid dendritic cells were consistently present, serving as endogenous positive control. 


\section{Discussion}

Monoclonal antibody to Hector Battiforamesothelial epitope-1 (HBME-1) has been raised against a biochemically unknown epitope presented in the membrane of the mesothelial cells, outlining their microvillous surface in a so-called thick membrane pattern. The HBME-1 represents a mouse monoclonal antibody of IgM-class, which immunoglobulin class is suggestive for a carbohydrate determinant on a glycoprotein or polysaccharide antigen.

The HBME-1 shows reactivity in a rather broad range of epithelial and mesenchymal tumors, and represents a multipurpose immunohistochemical marker in surgical pathology. It can be utilized to differentiate epithelial mesothelioma from lung adenocarcinoma as well as to distinguish malignant thyroid tumors from benign thyroid lesions. Peritoneal and ovarian serous carcinomas also display HBME-1 positivity. In mesenchymal tumors, chordoma, synovial sarcoma, and chondrosarcoma demonstrate HBME-1 reactivity which can be employed in the diagnostic work-up of these neoplasms. Interestingly, most of these HBME1-positive tumors also display microvillous membrane projections.

The HBME-1-positive lymphocytes were constantly demonstrated in non-neoplastic lymph node, tonsil, bone marrow, and spleen. The positive cells were detected in low number, but in some hyperplastic splenic tissues, in up to $10 \%$ of red pulp lymphocytes, and a significant proportion of monocytoidB cells in toxoplasma lymphadenitis showed HBME-1 staining. Confocal microscopy with multicolor immunofluorescence staining confirmed that the HBME-1-positive lymphocytes represent B cells. The staining pattern of the HBME-1 and DBA.44 revealed overlapping features with the main exception of strong mantle zone staining found only with the DBA.44. The majority of the HBME- $1^{+}$lymphocytes displayed villous cell membrane, which cells may represent a unique B cell population. The relationship between these villous lymphocytes and interfollicular large B cells with stellate morphology, described in peripheral lymphoid tissue, is yet to be delineated.

Since the HBME-1-positive reactive lymphocytes can be found in the splenic red pulp and most of these cells show villous surface, one might expect the HBME-1 to be positive in B celllymphoproliferations with villous membrane projections, therefore HCL and its mimics were overrepresented in our first series (overall 189/427 cases). The HBME-1 staining of lymphoid tumor cells was exclusively detected in the B cell neoplasms, supporting that the HBME-1 reacts with an epitope on the B lymphoid cells. 
As we anticipated, the HBME-1 staining was high in the B celllymphoproliferations with villous cells, in our hands it showed a very high positivity rate $(95.8 \%)$ in the HCL, which was concordant with that of DBA.44 (97.2\%), and also with Annexin A1 (96\%) found in a recent study. Our study has established that the HBME-1 is at least as sensitive as but fairly more specific as the DBA.44 in detecting tumor cells of HCL, and it can be particularly useful in the differential diagnosis of indolent B cell lymphomas with confluent interstitial bone marrow involvement. Since the HBME-1 is widely applied in surgical pathology and can be part of the antibody inventory in most immunohistochemical laboratories, it constitutes a simple, inexpensive assay of the HCL, easily fitted in the diagnostic antibody panel for routinely fixed and processed samples.

As it requires special therapy, the HCL must be distinguished from HCL-v, SDRPL, SMZL, and from other indolent B celllymphoproliferations with predominant bone marrow and splenic involvement. The HBME-1 demonstrated significantly lower positivity rate in the HCL-v and SDRPL (38.5\% and 50\%, respectively) than in the HCL, usually with weaker staining intensity. The HCL-v is a rare clinico-pathological entity, showing morphologic and phenotypic overlaps with the HCL, some features intermediate between B cellprolymphocytic leukemia and HCL, and resistance to conventional HCL therapy. The HCL-v cases also exhibit overlap with SDRPL, both are defined as provisional entities in the 2008 update of the WHO Classification. Both shows diffuse splenic red pulp involvement and DBA.44 positivity and the latter was also confirmed by our study. The SMZL and SBCL cases revealed similar low HBME-1 positivity rate, since the great majority of our SBCL cases should comprise SMZL.

In non-splenic B cell lymphomas, the HBME-1 positivity was found in nodal and extranodal MZL cases in a similar low rate to SMZL, and was associated with monocytoidB cell morphology, which is parallel to the HBME-1 staining of reactive monocytoidB cells in hyperplastic lymph nodes.

Therefore, our first study has established that the HBME-1 reacts with a minor subpopulation of B cells exhibiting villous surface, which has not been described previously. We propose that the HBME-1 can be a useful marker in the diagnosis of HCL and other indolent lymphoproliferations of villous B lymphocytes.

In our second study, we have performed a consecutive analysis to evaluate the diagnostic usefulness of various HCL markers currently available for paraffin embedded sections including CD11c, CD25, and CD103 those represent standard constituents of diagnostic HCL 
panels in flow cytometry.To the best of our knowledge, this was the most comprehensive study so far, assessing HCL immunohistochemical markers in paraffin sections.

Upregulation of ANXA1 has been identified through gene expression profiling and, subsequently, it was introduced as a specific immunohistochemical marker of HCL. We found ANXA1 to be apparently $100 \%$ specific, sensitive and accurate, confirming the previous findings however this marker displayed an equivalently strong cytoplasmic and nuclear staining also in the granulocytic series, which might cause considerable difficulties in the assessment of BM samples. Therefore, additional immunohistochemical markers seem to be necessary to apply in the diagnosis of HCL.

Recently, BRAF-V600E mutation has been identified as a disease-defining genetic event in HCL. Subsequently, a novel BRAF V600E mutant-specific antibody has been reported as a highly specific immunohistochemical marker of HCL. Although this antibody is commercially available, due to its rather high price and the availability of a simple, sensitive and inexpensive genetic assay for BRAF V600E mutation, we decided not to include antibody VE1 in this study.

The V600E leads to constitutive activation of BRAF that phosphorylates MEK and ERK (the kinase phosphorylated by phospo-MEK). Immunochemical detection of constant ERK phosphorylation using a pERK-specific rabbit monoclonal antibody has been demonstrated as a sensitive diagnostic assay of HCL. Our results presented here strongly support the diagnostic value of the anti-pERK antibody, being negative in all but one non-HCL cases tested. This marker exhibited nuclear staining in activated non-neoplastic mesenchymal cells, which pattern could be disturbing in evaluating samples with low tumor burden.

CD103 is a cell surface glycoprotein representing $\alpha \mathrm{E}$ subunit of the heterodimer integrin $\alpha E \beta 7$. It has been discovered by raising monoclonal antibody B-ly7 directly against HCL. Since then, the anti-CD103 antibodies have been widely used in the diagnosis of HCL by flow cytometry. Recently, a novel monoclonal rabbit antibody has been described that can successfully detect neoplastic cells of HCL in formalin-fixed and paraffin-embedded samples. Our study confirmed its diagnostic utility, showing high sensitivity (100\%) in HCL, however, 75\% of HCL-v and about one third of other indolent splenic B cell lymphoma cases also revealed CD103 positivity. These results coincide with previous findings those have been described by flow cytometric applicationsor in paraffin-embedded samples. 
Detection of CD11c, the integrin $\alpha \mathrm{X}$ chain, has an established diagnostic value in HCL. It is widely used, in combination with CD25 and CD103, for flow cytometry in the diagnosis of HCL.Monoclonal antibody 5D11 was introduced for immunohistochemical staining of CD11c antigen in formalin-fixed and paraffin-embedded samples, and has been described as a successively sensitive and specific marker of HCL. In our hands, the CD11c/5D11 antibody showed 100\% sensitivity in HCL samples and also stained 1 case of HCL-V and 1 case of SDRPL with moderate membranous expression in most positive cases. Additionally, 3 of 11 B-CLL cases had weak and focal staining for CD11c in paraffin sections of BM samples, resulted in $85.4 \%$ specificity and $91.8 \%$ accuracy. Our results strongly support applicability of CD11c/5D11 antibody for the diagnosis of HCL in BM trephines fixed in Schaffer's fixative.

Since CD25, the human interleukin-2 receptor alpha chain, has been reported to be positive in HCL, it is a widely used flow cytometric marker for the diagnosis of HCL.Availability of monoclonal antibody 4C9 allows the qualitative identification of CD25 molecule in paraffinembedded tissue sections, and thus, this antibody is suitable for the diagnosis of HCL in BM trephine biopsies by immunohistochemical staining. Remarkably, to the best of our knowledge, ours is the first study so far using the CD25/4C9 antibody for the evaluation of HCL and its mimics. We found the CD25/4C9 to be a highly sensitive marker for HCL in BM trephine biopsy samples capable of distinguishing HCL and other small B cell lymphomas, including HCL-v, SMZL, SDRPL, and SBCL. Nevertheless, we observed CD25 positivity also in about half of the CLL and MCL cases, which finding is in accordance with the data reported previously. Apart from neoplastic cells of small B cell lymphomas, activated nonneoplastic B and T cells also show CD25 positivity which can be troublesome in evaluating BM samples with low lesional cell content, like in minimal residual disease.

After we demonstrated in our first study that HBME-1 reacts with a minor subpopulation of $\mathrm{B}$ cells exhibiting villous surface, our findings in the second investigation reinforced its usefulness in HCL as it showed identical results with the CD103/EPR4166(2) antibody. We propose that, HBME-1 represents a low cost immunohistochemical marker of HCL and its mimics, easily accessible in most laboratories. Nevertheless, inhomogeneous or partial staining, found in a proportion of HCL can restrain its applicability when the tumor cell burden is low.

Overexpression of cyclin D1 is a disease-defining event in MCL. The expression of cyclin D1 has been described also in the neoplastic cells of HCL, but it is not related to overt 
rearrangements within the BCL-1 locus. In accordance with the previous findings, we demonstrated here that cyclin D1 shows very high specificity and accuracy in HCL too. Since cyclin D1 displays nuclear staining with variable intensity, it might not be suitable to ascertain HCL cells in BM samples with low lesional cell content.

Anti-CD68 antibody KP1 is a widely used monocyte/macrophage marker. Although it is seldom used for this purpose, it has been described to be positive in HCL. In our study, we found the CD68/KP1 to be $100 \%$ sensitive for HCL, but was positive also in several non-HCL cases, in macrophages, and in myeloid cells which can limit its value in detecting sparse HCL cells.

Monoclonal antibody DBA.44 identifies a fixation-resistant B cell differentiation antigen, showing positivity in HCL. For years, the DBA.44 was considered to be a standard immunohistochemical stain for the diagnosis of HCL. According to our own observation, this marker cannot differentiate between HCL and its mimics, since most splenic small B cell lymphoma cases $(73.3 \%)$ found to be DBA.44-positive. Moreover scattered, non-neoplastic lymphocytes can be frequently detected with this antibody in reactive conditions, so identifying residual tumor cells with this marker alone is cumbersome. However, all B-CLL, LPL/WM and MCL cases were consistently DBA-44-negative.

Enzyme histochemical detection of TRAP activity has been known as a conventional marker of HCL. Immunohistochemical detection of TRAP has been reported to be highly sensitive in HCL, in particular in combination with DBA.44. In this study, anti-TRAP immunostaining was positive in all cases of HCL and almost all non-HCL small B cell lymphomas, providing very low diagnostic value in discriminating these neoplasms.

Expression of T-bet, a T cell-associated transcription factor, has been described in the neoplastic cells of HCL and suggested to be an effective marker of minimal residual disease. In our study, the T-bet exhibited $100 \%$ positivity rate in HCL, but almost all small B cell lymphomas, both splenic and non-splenic cases, furthermore reactive $\mathrm{T}$ cells also demonstrated T-bet positivity, which appears to limit its discriminating value. Nevertheless, its homogeneous strong nuclear staining in HCL is easily recognizable and can be a useful marker in follow-up biopsies, especially in the diagnosis of minimal infiltration by HCL in BM trephines. 
CD200 (OX-2) was described to be consistently expressed on hairy cells. Yet, its positivity has been widely detected also in other B cell-derived neoplasms. Our study reassured these findings as the CD200 was found to be positive in all HCL and in the majority of other small B cell lymphoma cases. Our findings confirmed its value in the differential diagnosis of BCLL and MCL as we showed positivity in all B-CLL and consistent negativity in MCL samples.

Detection of CD123 ( $\alpha$-chain of interleukin-3 receptor) antigen was reported to assist the diagnosis of HCL in flow cytometry. There are monoclonal antibodies available against fixation resistant epitope of CD123 which can facilitate its demonstration in paraffinembedded HCL samples. In a pilot study, we have tested two distinct monoclonal mouse antiCD123 antibodies (7G3 and BR4MS), but neither of them has given sufficient sensitivity in HCL cells. Although a blend of these two antibodies resulted in some improvement (not shown), the CD123 still showed the overall lowest sensitivity in our HCL samples.

Detection of minimal infiltration, including minimal residual disease of HCL in paraffinembedded BM biopsy specimens can be of great diagnostic value. ANXA1, being the most specific HCL marker in our series too, displays a strong cross-reactivity with granulocytic series. Phospho-ERK, CD11c, CD25, HBME-1, CD68/KP1, DBA-44, T-bet, CD200, and TRAP exhibited positivity in various non-neoplastic cells which can be troublesome when evaluating low tumor cell content. Inhomogeneous staining with HBME-1, cyclin D1, and CD123 in a proportion of HCL cases can also be a limiting factor. Considering intensity, staining pattern, and distribution, CD103 and T-bet, in combination with CD20, seem to be the most promising markers for follow-up biopsies with minimal infiltration of HCL in BM trephine biopsies, but their usefulness is yet to be delineated.

In conclusion, our results reassured high specificity of ANXA1 and pERK as well as the diagnostic value of standard HCL markers of CD11c, CD25, and CD103 also in paraffinembedded BM samples. Additional markers, including HBME-1, cyclin D1, CD200, and Tbet also represent valuable tools in the differential diagnosis of HCL. As an optimal panel with high specificity and low cost, we propose the diagnostic application of CD20, ANXA1, pERK, CD103, and HBME-1 antibodies for paraffin-embedded BM samples suspicious for HCL. 


\section{Original observations}

1. Our study is the first that demonstrates the applicability of monoclonal antibody HBME-1 in hematopathology.

2. Ihave demonstrated that the HBME-1 reacts with a minor subset of non-neoplastic and malignant lymphoid cells with villous surface.

3. With multicolor immunofluorescence staining I have demonstrated that these HBME1 positive villous lymphocytes are of B cell origin.

4. I have verified that HMBE-1 may have the potential for use as a marker for HCL and is more reliable than DBA.44 which is not specific for HCL.

5. I have analyzed all but one available immunohistochemical markers ever reported to be useful in the diagnosis of HCL. To the best of my knowledge this is the most comprehensive immunohistochemical study concerning HCL.

6. To the best of my knowledge, mine is the first study so far using the CD25/4C9 antibody for the evaluation of HCL and its mimics. I have found the CD25/4C9 to be a highly sensitive marker for HCL in BM trephine biopsy samples capable of distinguishing HCL and other small B cell lymphomas, including HCL-v, SMZL, SDRPL, and SBCL.

7. I have demonstrated that CD25, CD11c and CD103 antibodies, which are routinely used in diagnosis of HCL by flow cytometry specimens, function trustworthily by formalin fixed and paraffin embedded sections as well.

8. I have proposed an optimal panel of antibodies (CD20, ANXA1, pERK, CD103, and HBME-1) with very high sensitivity and specificity and acceptable cost for paraffinembedded BM samples suspicious for HCL. 


\section{Acknowledgements}

I thank Dr. LászlóKrenácsand Dr. EnikőBagdifor assuring me the conditions of my work and for their helping with ideas and comments upon all the works being mentioned in the thesis. I am also grateful to ÁgnesDudás for her indispensable technical assistance. This work is dedicated to my mother and to my daughters, who saw me through my hard moments. 